\title{
The Unique Natural Number Set and Distributed Prime Numbers
}

\author{
Alabed $\mathrm{TH}^{1 *}$ and Bashir $\mathrm{MB}^{2}$ \\ ${ }^{1}$ Computer Science, Shendi University, Shendi, Sudan \\ ${ }^{2}$ Shendi University, Shendi, Sudan
}

\begin{abstract}
The Natural number set has a new number set behind three main subset: odd, even and prime which are unique number set. It comes from a mathematical relationship between the two main types even and odd number set. It consists of prime and T-semi-prime numbers set. However, finding ways to understand how prime number was distributed was ambiguity and it is impossible, this new natural set show how the prime number was distributed and highlight focusing on T-semi-prime number as a new sub natural set.
\end{abstract}

Keywords: Prime number; T-semi-prime number; Unique number set

\section{Introduction}

The Unique natural number set is sub natural number set which consist of prime and T-semi-prime numbers. T-semi-prime is natural number divided by one or more prime numbers. Although Prime numbers is a widely covered field by current and historical researchers, still main questions about it unsolved. This study attempts to provide a new way to know how primes number distribution throughout the infinite natural number. The definition of a prime is rather simple but the problems (headaches) its investigation has given to number theorists have been enormous. It has a present by earliest society record which comes from the Greeks and Euclid's Elements in 13 books which written by Euclid. It was included important theorems about primes [1]. The first ten prime numbers can be listed: $2,3,5,7,11,13,17,19$, 23 and 29 . In the literature, many papers cover specific topics widely but there is a lack of interesting overviews of the simplicity of early proofs, questions asked, answered and unsolved in the literature for the mathematicians to explore. Although prime number areas are old, still a number of questions unanswered. One of these questions is the twin prime conjecture is the first problem from a large list of unanswered questions. It looks at the distribution of prime numbers such as 11 and 13 that are separated by a single even integer. The question is simple, do the pairs of prime numbers continue on indefinitely [2]? Further questions about the separation between primes are also unanswered such as are there an infinite many prime number divided by 3 composite numbers [3]. Another unproven question is Goldbach Conjecture which surmised as every even integer can be broken into the summation of two prime numbers or 1 and all odd integers above 5 can be expressed as a sum of 3 prime numbers [4]. In addition, Euler used the prime series, furthermore it showing that primes are infinitely [5]. Addition unproven Conjecture was Riemann's Conjecture [3]. who discovered there was a correlation between the zero values of the zeta function and prime numbers and Riemann Zeta Function is still unproven [3]. But Euclid's proof that, the prime numbers are infinitely prime numbers in the proposition 20 of book IX of Euclid's Elements states [6].

On the last years, there are real life prime number applications. One of these applications is Encryption. It uses prime numbers and the almost unworkable job of factorizing exceptionally large numbers to encrypt, which is to jumble important information such as credit card numbers into unusable information for any other then the intended receiver. Also, Prime numbers are no longer chained to purely mathematical sciences and it has been used prime number within theoretical physics to try and produce a theory that ties together prime numbers with quantum chaos theory [3]. However, researchers were made a big effort to find algorithm which can describe how prime number are distributed, still distribution of prime numbers throughout a list of integers leads to the emergence of many unanswered and partially answered questions [7]. As a result of deep leaning on natural number set and the role of no thing happen randomly, this paper proposed algorithm will prove that prime number set are distributed according the mathematical formula.

\section{The Unique Number Set}

By looking into history of natural number, Euclid's in book7 definition 6 is "An even number is that which is divisible into two parts" [1] a whole number is even if it can be divided into two "level" or "uniform" amounts and odd is uneven things. As a result, by adding 1 to even number will get odd number. This mathematical relations are very simple and known but if we adding a new mathematical concept to this relation between odd and even number, it will get a new natural set. Always looks to relation number with number but if we look to the relation of one even number relate with two odd numbers and this relation is adding, it will bring natural numbers which solve the problem of distributed prime numbers. The unique numbers set is a partial set of natural set. Which has two type of number, prime and T-semi-prime? Prime are known from introduction but T-semi-prime is a new number type which defined as a natural number divisible by themselves, one or more prime numbers and 1 . The listed of first T-semi-prime are 25, 35 and 49 . These numbers from unique number set.

\section{Theorem}

Suppose that $n$ is even numbers and $n \geq 2,2 n-1,2 n+1$ are odd numbers and by making a mathematical procedure which is $(n+(2 n-$

*Corresponding author: Alabed TH, Computer Science, Shendi University, Shendi, Sudan, Tel: 24915566 2100; E-mail: tawalabed@gmail.com

Received August 21, 2017; Accepted October 14, 2017; Published October 24 2017

Citation: Alabed TH, Bashir MB (2017) The Unique Natural Number Set and Distributed Prime Numbers. J Appl Computat Math 6: 368. doi: 10.4172/21689679.1000368

Copyright: @ 2017 Alabed TH, et al. This is an open-access article distributed under the terms of the Creative Commons Attribution License, which permits unrestricted use, distribution, and reproduction in any medium, provided the original author and source are credited. 
Citation: Alabed TH, Bashir MB (2017) The Unique Natural Number Set and Distributed Prime Numbers. J Appl Computat Math 6: 368. doi: 10.4172/2168-9679.1000368

$1)=A)$ and $(n+(2 n+1)=B)$. Then the $A$ and $B$ are unique numbers.

$$
\begin{aligned}
& B=(n+(2 n+1) \\
& B=(n+(2 n+1)
\end{aligned}
$$

\section{Proof}

Let $\mathrm{n}$ is even and $2 \mathrm{n}-1,2 \mathrm{n}+1$ be odds. By hypnosis's, $\mathrm{n}+(2 \mathrm{n}-1)=\mathrm{A}$ and $n+(2 n+1)=B$. then the definition of unique set mathematical formula tell us that $\mathrm{A}$ and $\mathrm{B}$ be a unique numbers.

Basis: $\mathrm{n}=2$.

$$
\mathrm{n}+(2 \mathrm{n}-1)=2+3=5
$$$$
\mathrm{n}+(2 \mathrm{n}+1)=2+5=7
$$

5 and 7 are prime number and be unique set

Induction: Let $\mathrm{n}=4,6,8,10,12,14,16,18$ and 20 .then

$$
\begin{aligned}
& 4+7=11 \\
& 4+9=13 \\
& 6+11=17 \\
& 6+13=19 \\
& 8+15=23 \\
& 8+17=25 \\
& 10+19=29 \\
& 10+21=31 \\
& 12+23=35 \\
& 12+25=37 \\
& 14+27=41 \\
& 14+29=43 \\
& 16+31=47 \\
& 16+33=49 \\
& 18+35=53 \\
& 18+37=55 \\
& 20+39=59 \\
& 20+41=61 .
\end{aligned}
$$

Above all we note that, all results are constructing unique set because its entire prime or T-semi-prime likes 25, 35, 49 and 55. So, we can apply it using simple algorithm and simple code to generate a lot of number of prime and T-semi-prime numbers.

\section{Algorithm}

Before Problem: find n unique number which content two type, prime or T-semi-prime.

Step1: start.

Step2: output "the unique number set".
Step3: input even number $\mathrm{n}$.

Step4: output "inserts" $n$.

Step5: for i to $\mathrm{n}$ do

Step6: input $n$

Step8: A $\mathrm{n}+(2 \mathrm{n}-1)$

Step9: B $\mathrm{n}+(2 \mathrm{n}+1)$

Step10: output "unique number set are" \& A, B

Step11: stop.

\section{Java Code Details}

public class math \{

public static void main(String[] args) \{

// TODO Auto-generated method stub

for $($ int $\mathrm{i}=2 ; \mathrm{i}<10000 ; \mathrm{i}=\mathrm{i}+2)\{$

int $\mathrm{a}=\mathrm{i}+\left(2^{\star} \mathrm{i}-1\right)$;

int $b=i+\left(2^{*} i+1\right)$;

System.out.println( $(\mathrm{a}+$ " "+b);

\} .

\section{Conclusion}

The unique Natural number set is a new natural number set behind three main subset: odd, even and prime. It comes from a mathematical relationship between the two main types even and odd number set. It consists of prime and T-semi-prime numbers set. These unique number set contain prime numbers and $\mathrm{T}$-semi-prime numbers which divided by 1 , themselves and prime numbers. Its show how these prime numbers was distributed and highlight focusing on T-semi-prime number as a new sub natural set.

\section{Acknowledgments}

Thanks to $\mathrm{D} /$ mohammed Bakri for assistance with the preparation of the text. Thanks to Abdalfattah for helping to implement code.

\section{References}

1. Heath TL (1956) The thirteen books of Euclid's Elements. Courier Corporation.

2. Oladejo N, Adetunde I (2009) A numerical test on the Riemann hypothesis with applications. Journal of Mathematics and Statistics 5: 47.

3. Tularam G (2011) The importance of numbers and the need to study primes: The prime questions. Journal of Mathematics and Statistics 7: 262-269.

4. Burton DM (2006) Elementary number theory.Tata McGraw-Hill Education.

5. Gautschi W(2008) Leonhard Euler: His life, the man, and His works. SIAM review 50: 3-33

6. Siu M, Tzanakis C (2004) The role of the history of mathematics in mathematics Education. Mediterranean Journal for Research in Mathematics Education, p: 3.

7. Stein W, Mazur B (2007) What is Riemann's Hypothesis. Lecture presented at Summer Institute for Mathematics at the University of Washington. 